\section{Response to: 'Is primary Sjögren's syndrome an orphan disease? A critical appraisal of prevalence studies in Europe' by Cornec and Chiche}

We thank Divi Cornec and Laurent Chiche $^{1}$ for their valuable comments on our article. ${ }^{2}$ We think that the majority of remarks they made were reasonable and helpful for a deep understanding of the prevalence of primary Sjögren's syndrome (pSS). Due to the great differences between a population-based study and sample survey, it is obvious that a high heterogeneity $\left(\mathrm{p}<0.001\right.$ for $\mathrm{Q}$ statistic, $\left.\mathrm{I}^{2}=98.95 \%\right)$ could be observed across these articles when conducting a meta-analysis. According to the definition of a rare disease, the total prevalence rate from our study did show that pSS is not a rare disease (one case in 1644). But the prevalence rate was one case per 2304 persons when only considering a population-based study, suggesting pSS is a rare disease.

Among the included studies in our paper, pSS patients from sample survey were mainly found through the questionnaire. In this process, several biases including non-response bias, coverage bias and selection bias, and so on influence the accurate prevalence rate of pSS in a defined region or a target population. However, a population-based study is often used from the administrative database or hospital medical records. Then, the population-based study could avoid these biases and provide a more accurate prevalence rate of pSS. When conducting the systematic review and meta-analysis, it is impossible to completely exclude the effect of these biases on the results. This is also a limitation of this study which was present in our article. ${ }^{2}$ Then, we analysed the differences in prevalence rate through a subgroup analysis, and the results also demonstrated that prevalence rate was greatly different between those two types of studies (43.03 cases per 100000 inhabitants vs 282.35 cases per 100000 inhabitants). The results from subgroup analysis showed that pSS is a rare disease (population-based study), but also not a rare disease (sample survey). Of course, the results from the population-based study may be more accurate and reliable. Except for the study design, the sample sizes also vary greatly among these included studies. But the results from meta-regression show that study design contributes to the high heterogeneity rather than sample sizes.

In all, $50 \%$ of included studies concerning the prevalence rate of pSS were based on the AECG 2002 diagnostic criteria. AECG 2002 criteria is the most widely accepted classification criteria currently, which was published by the AmericanEuropean Consensus Group in 2002. ${ }^{3}$ The pooled prevalence rate originated from studies using AECG 2002 was 73.57 per 100000 inhabitants, but most studies were designed as sample survey. The studies using ICD diagnoses gave a relatively low estimated prevalence rate of 38.60 , but most studies using ICD code were designed as a population-based study. Therefore, we think the study design may be a main reason for these differences.

There are 11 studies from Europe, which gave a pooled prevalence rate of 71.22 cases per 100000 inhabitants (one case per 1404 persons). Based on these data, pSS may be not a rare disease in Europe. The pooled prevalence rate from European population-based studies was 45.47 cases per 100000 in figure $1 \mathrm{~A}$. When only including studies that were designed as population-based studies and using AECG 2002, the meta-analysis also gave a pooled prevalence rate of 48.99 cases per 100000 in figure $1 \mathrm{~B}$. All these results suggest that pSS is a rare disease. Due to the high accuracy of the population-based study, the concept that pSS is a rare disease in Europe could be acceptable, to some extent.

Of note, the case-finding method, case-ascertainment method and study design are important indexes to evaluate the quality of an epidemiological study. The population-based study using definition diagnostic criteria has a better quality than others. This type of epidemiological study could provide accurate data, therefore, and needs to be conducted in different regions to assess whether pSS is a rare disease.
Figure 1 Pooled prevalence rate for primary Sjögren's syndrome per 100000 inhabitants across European studies. (A) All European population-based study; (B) All European population-based study using American-European Consensus Group 2002.

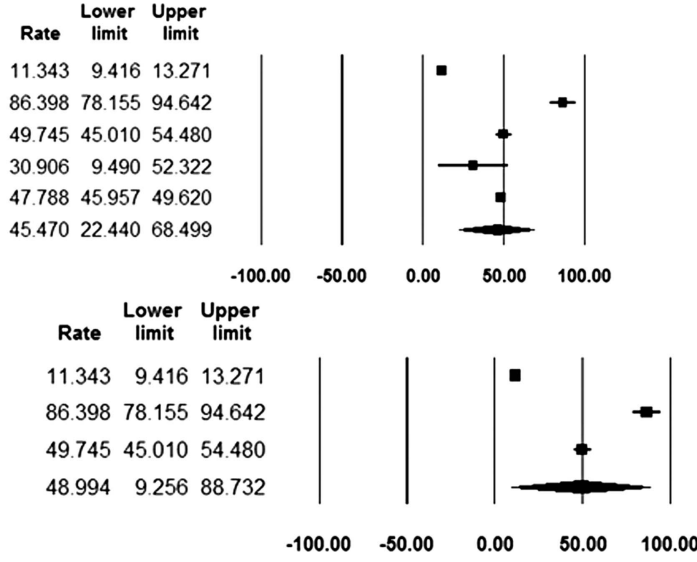


Baodong Qin, ${ }^{1}$ Jiaqi Wang, ${ }^{2}$ Zaixing Yang, ${ }^{1}$ Yan Liang, ${ }^{1}$ Renqiang Zhong ${ }^{1}$

${ }^{1}$ Department of Laboratory Diagnostics, Chang Zheng Hospital, Second Military Medical University, Shanghai, China

${ }^{2}$ Department of Stomatology, Changzheng Hospital, Second Military Medical University, Shanghai, China

Correspondence to Baodong Qin, Department of Laboratory Diagnostics, Chang zheng Hospital, Second Military Medical University, Shanghai 200003, China; qbdchn11@126.com

\section{Competing interests None.}

Provenance and peer review Commissioned; internally peer reviewed.

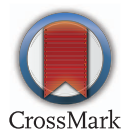

To cite Qin B, Wang J, Yang Z, et al. Ann Rheum Dis 2015;74:e26.

Accepted 28 October 2014
Published Online First 11 November 2014

\section{S Linked}

- http://dx.doi.org/10.1136/annrheumdis-2014-206860

Ann Rheum Dis 2015;74:e26. doi:10.1136/annrheumdis-2014-206885

\section{REFERENCES}

1 Cornec D, Chiche L. Is primary Sjögren's syndrome an orphan disease? A critical appraisal of prevalence studies in Europe. Ann Rheum Dis 2015; 74:e25.

2 Qin B, Wang J, Yang Z, et al. Epidemiology of primary Sjögren's syndrome: a systematic review and meta-analysis. Ann Rheum Dis Published Online First: 17 Jun 2014. doi:10.1136/annrheumdis-2014-205375

3 Vitali C, Bombardieri S, Jonsson R, et al. Classification criteria for Sjogren's syndrome: a revised version of the European criteria proposed by the American-European Consensus Group. Ann Rheu Dis 2002;61:554-8. 